Research Paper

\title{
A Long Non-coding RNA Lnc712 Regulates Breast Cancer Cell Proliferation
}

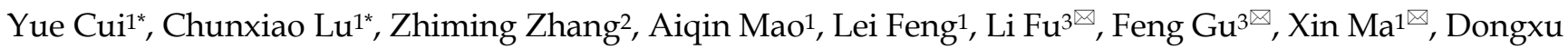 \\ $\mathrm{He}^{2 \bowtie}$ \\ 1. Wuxi School of Medicine, Jiangnan University, Wuxi, China. \\ 2. School of Food Science and Technology, Jiangnan University, Wuxi, China. \\ 3. Department of Breast Cancer Pathology and Research Laboratory, State Key Laboratory of Breast Cancer Research, Cancer Institute and Hospital, Tianjin \\ Medical University, Tianjin, China. \\ * These authors contributed equally to this study
}

$\square$ Corresponding authors: Dongxu He, Ph.D. School of Food Science and Technology, Jiangnan University, Wuxi, China. Email: hedongxu@jiangnan.edu.cn; Xin Ma, Ph.D. Wuxi School of Medicine, Jiangnan University, Wuxi, China. Email: maxin@jiangnan.edu.cn; Feng Gu, M.D. Department of Breast Cancer Pathology and Research Laboratory, State Key Laboratory of Breast Cancer Research, Cancer Institute and Hospital, Tianjin Medical University, Tianjin, China. Email: fenggumayo@163.com; Li Fu, M.D. Department of Breast Cancer Pathology and Research Laboratory, State Key Laboratory of Breast Cancer Research, Cancer Institute and Hospital, Tianjin Medical University, Tianjin, China. Email: fulijyb@hotmail.com

(C) The author(s). This is an open access article distributed under the terms of the Creative Commons Attribution License (https://creativecommons.org/licenses/by/4.0/). See http://ivyspring.com/terms for full terms and conditions.

Received: 2019.05.06; Accepted: 2019.07.29; Published: 2020.01.01

\begin{abstract}
Great quantity of intergenic noncoding RNAs (IncRNAs) have been identified in the mammalian genome and involved in various biological processes, especially in the development and metastasis of cancer. In this study, we identified one IncRNA, IncRNA NONHSAT028712 (Lnc712), was highly expressed in breast cancer cell lines and tissues based on microarray screening. Knockdown of Lnc712 largely inhibited breast cancer cell proliferation. Mechanistically, Lnc712 bound specifically to heat-shock protein 90 (HSP90). Interaction between Lnc712 and HSP90 is required for HSP90 binding to cell division cycle 37 (Cdc37). The Lnc712/HSP90/Cdc37 complex regulated cyclin-dependent kinase 2 (CDK2) activation and then triggered breast cancer cell proliferation. In summary, our results identified a new IncRNA regulate breast cancer proliferation though interaction with HSP9O.
\end{abstract}

Key words: Long non-coding RNAs, Breast Cancer, HSP90, CDK2

\section{Introduction}

Breast cancer is one of the most common malignant tumors among women [1]; it is the fifth leading cause of cancer-related deaths worldwide, with an increasing incidence annually [2]. Breast cancer is a heterogeneous carcinoma, and both genetic and epigenetic changes contribute to its initiation and development. However, the underlying molecular and biological functions correlated with breast cancer are still unclear. Consequently, finding therapeutic targets is a major challenge.

Studies suggest that protein-coding genes account for $<2 \%$ of the human genome, whereas the vast majority of transcripts are non-coding RNAs [3, 4]. Long non-coding RNAs (lncRNAs), ranging from
200 nucleotides to $100 \mathrm{~kb}$, constitute the largest proportion of ncRNAs. LncRNAs regulate the transcription process by interacting with RNA-binding proteins, co-activating transcription factors, and repressing the promoters of target genes [5-7]. It has been suggested that lncRNAs play key roles in tumorigenesis [8].

In this study, we identified the novel function of one lncRNA, NONHSAT028712 (Lnc712), and showed that it plays a role in breast cancer cell proliferation. Up-regulation of Lnc712 was involved in breast cancer proliferation via the HSP90-Cdc37CDK2 pathway. 


\section{Materials and methods}

\section{Microarray}

The microarray profiling was conducted in the laboratory of OE Biotechnology Co. (Shanghai, China). RNA was separately extracted from MCF-7/ADM and MCF-7 cells using the acid-phenol and chloroform method. Cyanine-3-CTP-labeled cRNA was obtained using a Quick Amp labeling kit (Agilent Technologies, Santa Clara, CA, USA) and then purified with an RNeasy Mini kit (Qiagen, Valencia, CA, USA). The labeled cRNAs were then hybridized onto Agilent-062918 OE Human lncRNA Microarray V4.0 028004 (Agilent Technologies), which is a Custom Gene Expression Array for OE Biotechnology Co. and detects 46,506 lncRNAs. After washing, the arrays were scanned with an Agilent scanner (G2505C).

\section{Cells and Cultures}

Human breast epithelial cells (MCF-10A; American Type Culture Collection (ATCC)), the human breast cancer cells MDA-MB-231 and MCF-7 (ATCC), and long-term adriamycin [9]-treated MCF-7 cells (MCF-7/ADM) were cultured in Dulbecco's modified Eagle's medium (Gibco, Gaithersburg, MD, USA) supplemented with $10 \%$ fetal bovine serum (Sijiqing, Hangzhou, China), $100 \mu \mathrm{g} / \mathrm{mL}$ penicillin, and $100 \mathrm{U} / \mathrm{mL}$ streptomycin (Beyotime, Shanghai, China) at $37^{\circ} \mathrm{C}$ in a humidified atmosphere with $5 \%$ $\mathrm{CO}_{2}$. MCF-7/ADM cells were generated by treating MCF-7 cells with stepwise increasing concentrations of ADM over 8 months.

\section{RNA extraction and RT-qPCR analysis}

Total RNA was extracted with TRIzol according to the manufacturer's instructions (Invitrogen, Carlsbad, CA, USA). RNA was precipitated with GlycoBlue (AM9516, Ambion, Waltham, MA, USA) and dissolved in diethylpyrocarbonate-treated water. Samples of $1 \mu \mathrm{g}$ of DNase-treated RNA were reverse-transcribed using a PrimeScript RT reagent kit (RR047A, Takara, Shiga, Japan). qPCR was performed using SYBR Green mix (RR890A, Takara) with the cycling conditions $95^{\circ} \mathrm{C}$ for 30s followed by 40 cycles of $95^{\circ} \mathrm{C}$ for $5 \mathrm{~s}$ and $60^{\circ} \mathrm{C}$ for $30 \mathrm{~s}$.

LncNONHSAT028712: forward, 5'-AAATACCT CACCCTCATCTATACCAAC-3'; reverse, 5'-TTTCC CGTTGCCATTGAT-3'.

CDK2 (cyclin-dependent kinase 2): forward, 5'-CGCTTGTTAGGGTCGTAGTG-3'; reverse, 5'-AG ATTGACCAGCTCTTCCGG-3' .

GAPDH: forward, 5'-CAAGAAGGTGGTGAAG CAGG-3'; reverse, 5'-TCAAAGGTGGAGGAGTGG GT-3'.

\section{Patients and specimens}

For fluorescence in situ hybridization (FISH) validation of Lnc712, 10 fresh breast cancer and paired non-cancer tissue samples were collected at the Tianjin Tumor Hospital from 2017 to 2018. Inclusion criteria were patients with primary breast cancer, having tumor stage I-IV, and surgery was the initial treatment approach. Informed consent for the use of samples was given by all patients, and the study was approved by the Ethics Committee of the Institute of Basic Medical Sciences, Chinese Academy of Medical Sciences.

\section{Fluorescence in situ hybridization}

FISH was performed using the lncRNA FISH Probe Mix kit (Ribobio, Guangzhou, China). Briefly, sections were deparaffinized, dehydrated in $100 \%$ ethanol, and dried. Slides were incubated with hydrogen peroxide for $30 \mathrm{~min}$ at room temperature (RT), then subjected to protease digestion for $20 \mathrm{~min}$ and dehydrated in an ethanol series. The prehybridization buffer was applied to a selected area on each slide, and incubated at $37^{\circ} \mathrm{C}$ for $2 \mathrm{~h}$. The slides were incubated with hybridization buffer for co-denaturation of 1 ncRNA and probe RNA at $37^{\circ} \mathrm{C}$ for $16 \mathrm{~h}$. After hybridization, the slides were washed with saline-sodium citrate buffer and then mounted in anti-fade solution with DAPI.

\section{RNA-protein pull-down assays}

Biotinylated lncRNAs were refolded in structure buffer [10 mM Tris $\mathrm{HCl}$, pH 7.0, $10 \mathrm{mM} \mathrm{MgCl}_{2}, 0.1 \mathrm{M}$ $\mathrm{KCl}$. The diluted RNAs were incubated at $95^{\circ} \mathrm{C}$ for 2 $\mathrm{min}$, put on ice for $3 \mathrm{~min}$, and left at RT for $30 \mathrm{~min}$. For pull-down incubation, lysates containing $1 \mathrm{mg}$ protein were pre-cleared with streptavidin beads and then incubated with $2 \mu \mathrm{g}$ biotinylated RNA and streptavidin beads for $1 \mathrm{~h}$ at RT. The beads were collected by centrifugation and washed three times with buffer II [ $50 \mathrm{mM}$ Tris $\cdot \mathrm{HCl}$, pH 7.0, $1 \mathrm{mM}$ EDTA, $100 \mathrm{mM} \mathrm{KCl}, 0.1 \%$ TritonX-100, 5\% glycerol, $1 \mathrm{mM}$ DTT]. RNA-associated proteins were eluted and resolved on SDS/PAGE followed by silver staining according to the manufacturer's instructions (Bio-Rad Laboratories, Hercules, CA).

\section{Reverse Pull-Down Assays}

Lysates of MCF-7/ADM cells were prepared as described for RNA pull-down assays[10]. Samples containing $1 \mathrm{mg}$ protein were precleared with protein A/G beads for $1 \mathrm{~h}$ at $4^{\circ} \mathrm{C}$. HSP90 or mouse IgG-specific antibody (5 $\mu \mathrm{g} / \mathrm{sample})$ was mixed with protein $A / G$ beads and incubated with cell lysate overnight at $4^{\circ} \mathrm{C}$. The captured RNAs were purified using Protease $\mathrm{K}$ and phenol/chloroform 
precipitation; extracted RNAs were then subjected to Northern blot analysis.

\section{Small-interfering RNA transfection}

Cells were transiently transfected with gene-specific or scrambled siRNA using Lipofectamine 2000 transfection reagent (ThermoFisher, Waltham, MA, USA) following the procedure recommended by the manufacturer as described previously [19]. Lnc712 was silenced using an individual siRNA targeting the sequence 5'-GACCAGATATCCCTCGCTT-3'; HSP90 was silenced using an individual siRNA targeting the sequence 5'-GGAACGTGATAAAGAAGTA-3'.

\section{Cell vitality assays}

Cells were plated into 96-well plates and grown overnight at $37^{\circ} \mathrm{C}$ under $5 \% \mathrm{CO}_{2}$. After transfection with siRNA for $48 \mathrm{~h}, 10 \mu \mathrm{l}$ MTT (3-(4,5-dimethylthiazol-2-yl)-2,5-diphenyltetrazolium bromide) was added to each well and incubated for 4$6 \mathrm{~h}$ at $37^{\circ} \mathrm{C}$. Then the medium was removed and 100 $\mathrm{ml}$ dimethyl sulfoxide was added to each well. The optical density (OD) was measured with a microplate reader (Bio-Rad Laboratories, Hercules, CA) at 570 $\mathrm{nm}$ to assess the relative number of surviving cells.

\section{Cell-cycle analysis}

Cells were seeded in 6-well plates and cultured in medium at $37^{\circ} \mathrm{C}$ under $5 \% \mathrm{CO}_{2}$. Forty-eight hours after transfection, the cells were trypsinized and washed twice with cold phosphate-buffered saline (PBS). Then the cells were fixed in $1000 \mu 170 \%$ ethanol for $2 \mathrm{~h}$ at $4^{\circ} \mathrm{C}$, followed by the addition of $500 \mu \mathrm{l}$ cell-cycle staining buffer (Beyotime, Nantong, China). After 30-min incubation at $37^{\circ} \mathrm{C}$, a flow cytometer (BD Accuri $^{\mathrm{TM}}$ C6, Mountain View, CA) was used to analyze the DNA content of the cells.

\section{Western blot}

Cells were lysed in RAPI (Sigma-Aldrich, St. Louis, MO, USA) containing $1 \%$ phenylmethylsulfony fluoride (Beyotime, Shanghai, China) and centrifuged for $15 \mathrm{~min}$ at $4^{\circ} \mathrm{C}$. Protein concentrations were then measured using a BCA protein assay kit (Beyotime). Proteins were separated on $12 \%$ gels using sodium dodecyl sulfate polyacrylamide gel electrophoresis. For immunoblots, the PVDF membrane carrying the transferred proteins was incubated at $4^{\circ} \mathrm{C}$ overnight with designated primary antibodies diluted in TBST buffer containing $0.1 \%$ Tween 20 and $5 \%$ bovine serum albumen (BSA). Immunodetection was accomplished using a horseradish peroxidase-conjugated secondary antibody and an enhanced chemiluminescence detection system (P1108, Beyotime). The antibodies anti-CDK2 (ab32147), anti-HSP90 (ab13492),
anti-Cdc37 (ab224831), and anti-ubiquitin (ab134953) were from Abcam, and anti-GAPDH (AP0063) was from Bioworld Technology Inc. (St. Louis Park, MN, USA). Images were analyzed using ImageJ. (National Institutes of Health, Bethesda, MD, USA).

\section{Co-immunoprecipitation}

Forty-eight hours after transfection, cells were harvested and lysed in RIPA base buffer [50 mM Tris $\mathrm{HCl}, \mathrm{pH} 7.4,137 \mathrm{mM} \mathrm{NaCl}, 0.25 \%$ sodium deoxy cholate, $4 \%$ protease inhibitor cocktail]. Antibodies were incubated overnight with cell lysates at $4^{\circ} \mathrm{C}$ on a rotating platform. Forty microliters of protein A/G plus-agarose magnetic beads (Santa Cruz Biotechnology) was incubated with cell lysates for $4 \mathrm{~h}$ at $4^{\circ} \mathrm{C}$, then the beads were washed three times with PBS and the bound proteins were eluted by boiling for 5 min with $50 \mu \mathrm{l}$ SDS sample buffer. Samples were then subjected to Western blot analysis.

\section{Animal experiments}

MDA-MB-231 cells were injected subcutaneously into the flanks of female BALB/c mice $\left(5 \times 10^{6}\right.$ cells/mouse). All mice were housed under air-filtered, pathogen-free conditions. Tumor volumes were estimated using the formula: volume $\left(\mathrm{mm}^{3}\right)=$ $3.14 \times(\text { width })^{2} \times$ length/ 6 . When the tumors reached $\sim 200 \mathrm{~mm}^{3}$, the animals were also injected once every 3 days at the tumor sites with Lnc712 siRNA or scrambled siRNA (5 nmol) as control.

\section{Immunofluorescence analysis}

After incubation with 5\% BSA, the slides were incubated with primary antibody overnight at $4^{\circ} \mathrm{C}$ in a humidified chamber, followed by the appropriate secondary fluorescence-labeled antibody (Invitrogen) for $3 \mathrm{~h}$ at room temperature. Nuclei were counterstained with DAPI (Beyotime). Images were captured on a Zeiss confocal microscope.

\section{Statistical analyses}

Results are presented as mean \pm SD. We assessed comparisons between groups using one-way ANOVA or Student's t-test. We performed the statistical analyses using Graphpad Prism 5.0 software. All statistical tests were two-sided, and $\mathrm{P}$ values $<0.05$ were considered to be statistically significant.

\section{Results}

\section{Lnc712 is up-regulated in breast cancer}

An lncRNA microarray was used to analyze the expression of lncRNAs in breast cancer cells. In order to identify lncRNAs involved in breast cancer cell proliferation, progression or chemoresistance development, we compared the lncRNA profiles from 
MCF-7 cells and MCF-7/ADM cells. MCF-7/ADM cells are generated by long-term treatment with adriamycin, and they have a greater ability to proliferate, metastasize and resistance to chemotherapeutic drugs than the parental control MCF-7 cells (MCF-7/WT) [11, 12]. Therefore, LncRNAs differentially expressed in MCF-7/ADM cells plays as candidates that regulates cancer proliferation, progression or chemoresistance. Among the 60 most differentially-expressed lncRNAs, NONHSAT028712 (Lnc712) was significantly higher in MCF-7/ ADM cells (Fig. 1a, also see raw microarray data in GSE81971), and the result was validated with RT-PCR (Fig. 1b). Interestingly, unlike other chemoresistance-related proteins (e.g. p-glycoprotein [13] and glutathione S -transferase P1 [14]) that expressed at a low level in chemosensitive cancer cells, RT-PCR results showed although Lnc712 was higher in MCF-7/ADM cells, it is also expressed in MCF-7/WT cells (Fig. 1b). Therefore, Lnc712 may
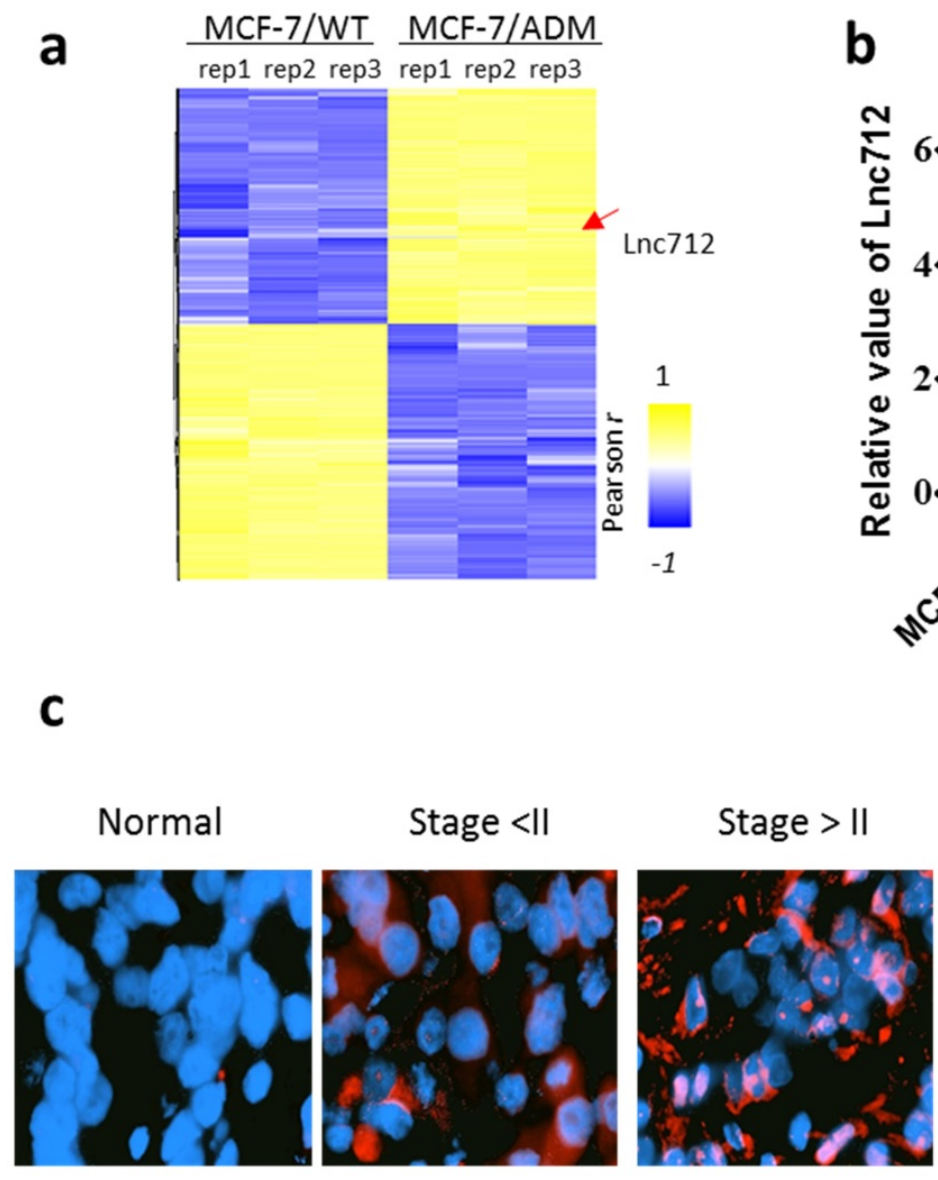

participate general signaling pathways involved in cancer proliferation and progression. To validate such assumption, we analyzed Lnc712 expression in another breast cancer cells line, MDA-MB-231 cells, and then compared Lnc712 expression in different breast cancer cells with that in non-tumorigenic epithelial cell line MCF-10A. As expected, the Lnc712 expression was lowest MCF-10A cells, while it was significantly higher in all three breast cancer cell lines, and it was highest in the more malignant MCF-7/ADM and MDA-MB-231 cells. Together, these results suggest a role of Lnc712 in breast cancer development.

We then validated the Lnc712 expression in clinical samples (Supplementary Table 1). RNA-FISH demonstrated that Lnc712 expression was significantly higher in breast cancer tissue than in adjacent normal tissue (Fig. 1c). Together, these results indicated that Lnc712 might relate to breast cancer progression.
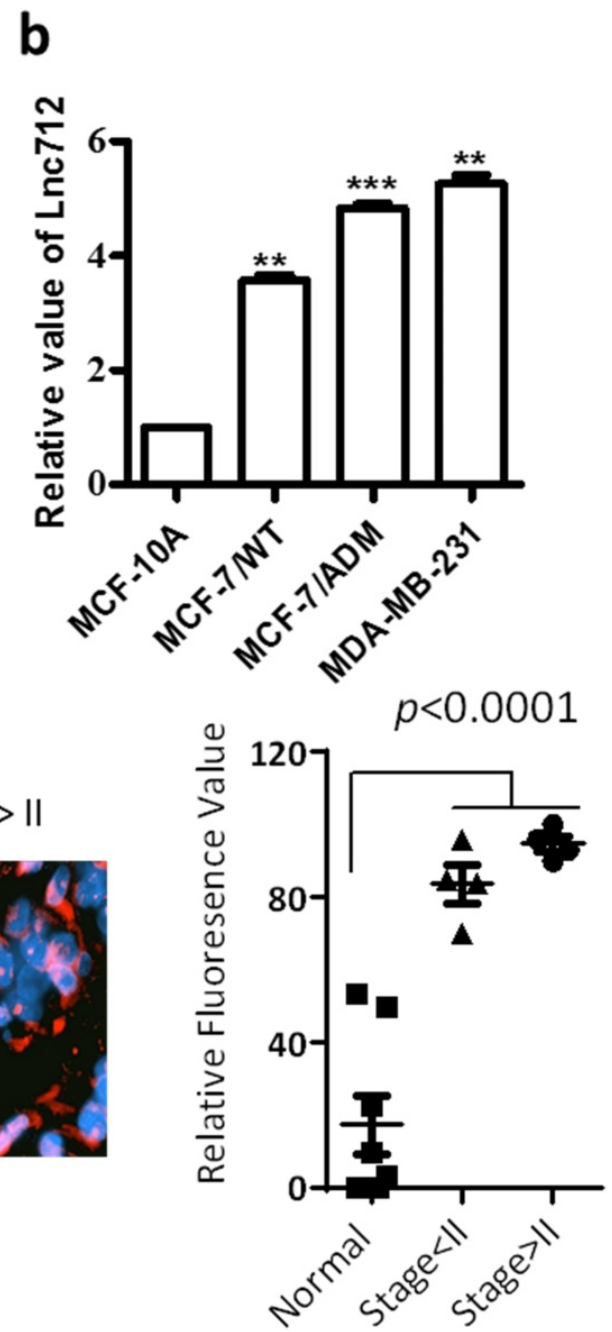

Figure 1. Lnc712 was up-regulated in breast cancer. (a) Hierarchical clustering of distinct IncRNAs in MCF-7/ADM and MCF-7/WT cells. Profiling was replicated 3 times. (b)RT-qPCR validated the expression of Lnc712 in normal breast epithelial cell and breast cancer cells, the values for MCF-10A cells were normalized to 1. Results are mean $\pm S D$. $* * P<0.01, * * * P<0.001$. (c) Lnc712 expression in breast cancer tissues and adjacent normal tissues were detected using FISH. $\mathrm{n}=10$. 


\section{Lnc7 12 is associated with heat-shock protein 90.}

RNA-FISH was then used to determine the localization of Lnc712, and it was found mainly in the cytoplasm (Supplementary Fig. 1), Suggesting this lncRNA may regulate cytosolic biological process, e.g. binding to cytosolic factors, rather than direct regulate gene expression. To understand the mechanism of action of Lnc712, we sought to identify intracellular Lnc712-binding factors using pull-down assays. Biotinylated Lnc712 (or a negative control RNA) was incubated with total protein extracts from MCF-7/ADM cells and pulled down with streptavidin beads to identify the associated proteins by silver staining (Fig. 2a). We chose four areas of bands for analysis by mass spectrometry, which later identified HSP90, EFZ2, and HMGB1 as Lnc712-binding proteins as confirmed by immunoblotting the proteins that were captured in the pull-down assay (Fig. 2b). Among these, HSP90 as a molecular chaperon that controls activation or ubiquitination of a client protein resulting in proteasomal degradation [15], were most specific. HSP90 is overexpressed in many cancers compared to its expression in normal tissues [16, 17]. HSP90-specific antibody also was able to enrich the endogenous Lnc712 in extracts from MCF-7/ADM cells (Fig. 2c).

To further understand the interaction between the Lnc712-HSP90 comp lex, three types of breast cancer cell were transfected with Lnc712 siRNA or HSP90 siRNA and the knockdown efficiency was confirmed by RT-qPCR and Western blot (Supplementary Fig. 2a and b). Since Lnc712 has a highly homologous gene that expresses GST3 on chromosome 11, we further validated the specificity of siRNA (Supplementary Fig. 2c). Interestingly, we found that Lnc712 expression was decreased in cells with HSP90-knockdown (Fig. 2d), suggesting that HSP90 is required for Lnc712 function and stabilization. On the contrary, knockdown of Lnc712 did not affect HSP90 expression (Fig. 2e and Supplementary Fig. 2d). Together, these results indicated that Lnc712 and HSP90 form a functional ribonucleoprotein complex.

\section{Lnc712-HSP90 regulates breast cancer cell proliferation via the CDK2 pathway}

HSP90 stabilizes a number of proteins required for tumor growth. Knockdown of HSP90 decreased the viability of MCF-7, MC-7/ADM, and MDA-MB-231 cells (Supplementary Fig. 3a). Similarly, we found that after Lnc712 expression was decreased by siRNA, the viability of MCF-7, MC-7/ADM, and MDA-MB-231 cells was markedly decreased (Fig. 3a). The decreased viability was due to cell-cycle arrest at G0/G1 (Fig. 3b and Supplementary Fig. $3 b$ ). These results suggest that Lnc712 is required for the function of HSP90 in regulating tumor growth.

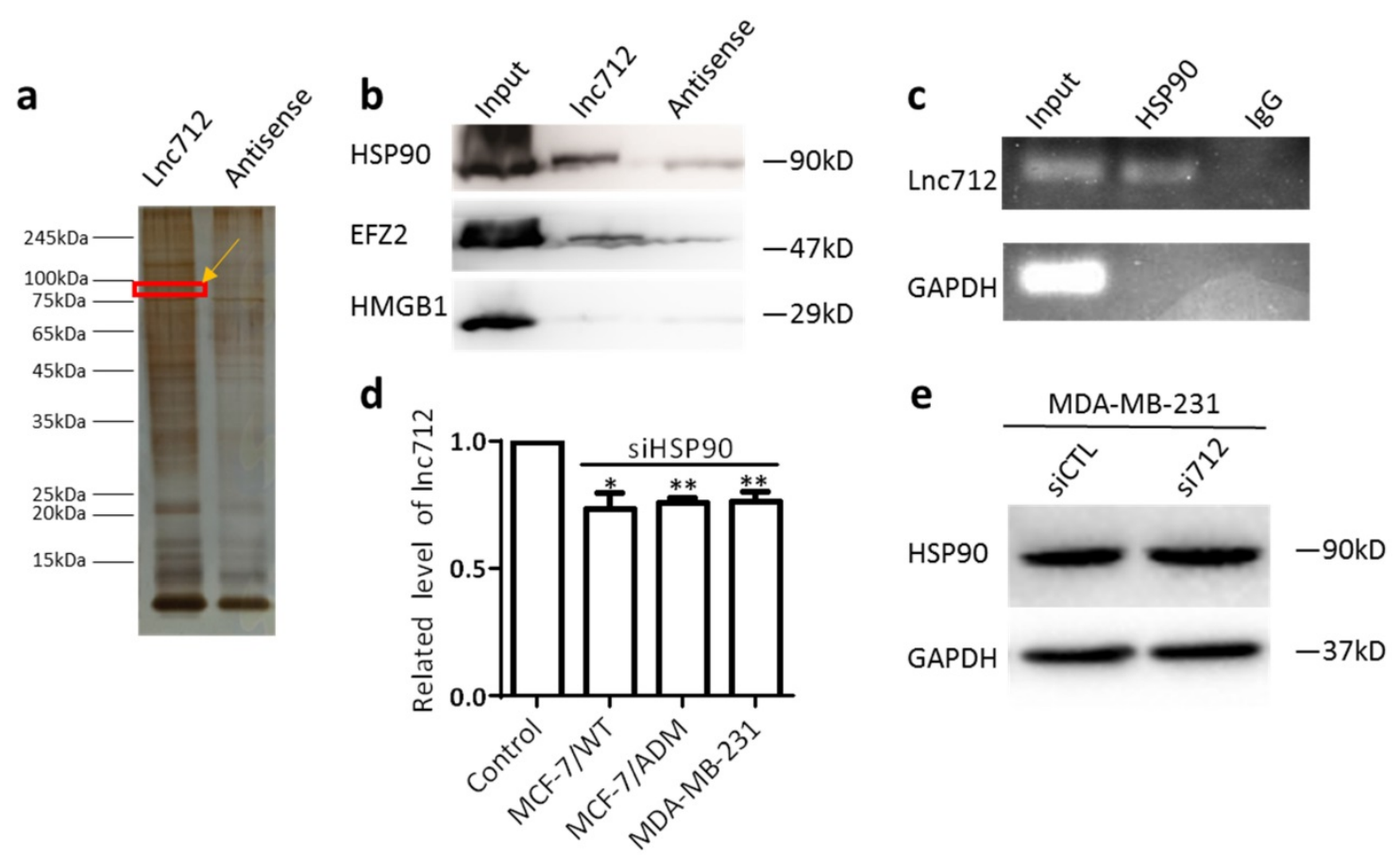

Figure 2. Lnc712 is associated with the HSP90 protein. (a)Silver staining of biotinylated Lnc712-associated proteins. (b)Western blotting of proteins from Lnc712 pull-down assays. (c) RIP experiments were performed using the HSP90 antibody to probe RNA from MDA-MB-231 cell extracts, and the levels of the coprecipitated RNAs were determined using qRT-PCR. (d )Lnc712 expression in HSP90 knockdown cells. Results are mean \pm SD. **P<0.01. (e)Western blot analysis of the effect of Lnc712 knockdown on HSP90 in MDA-MB-231 cells. 
a
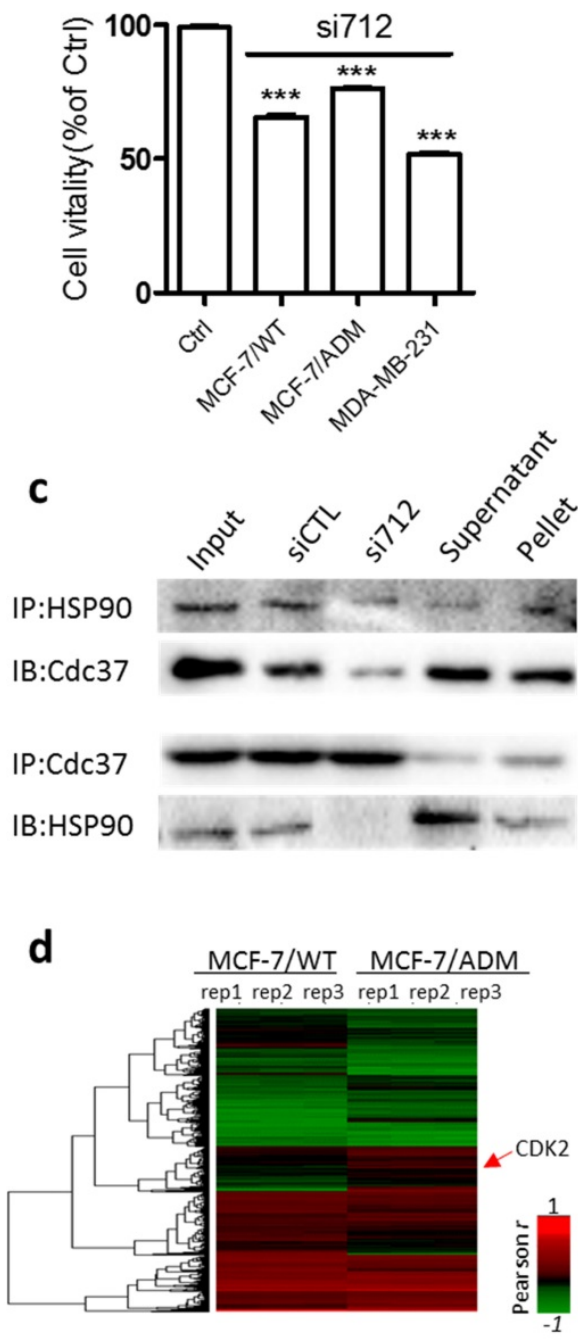

h

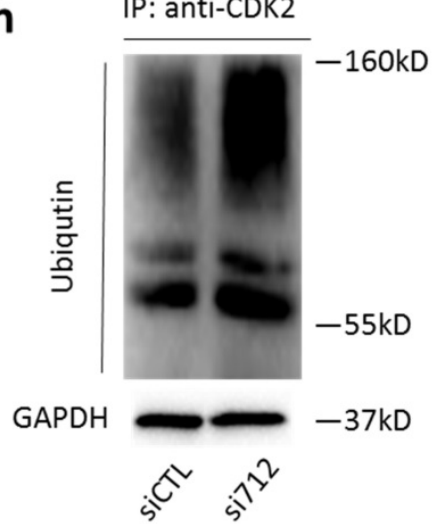

b

MDA-MB-231

SiCTL

$\operatorname{si712}$

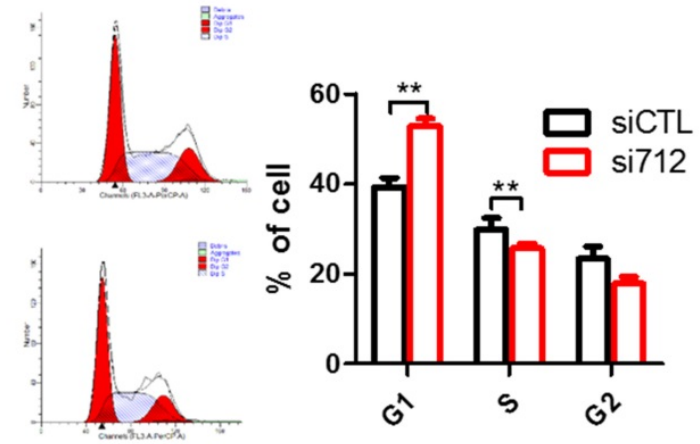

e

CDK2
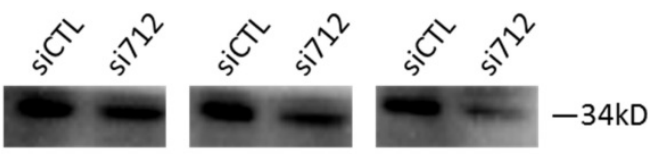

GAPDH
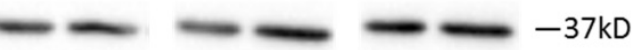

MCF-7/WT MCF-7/ADM MCA-MB-231
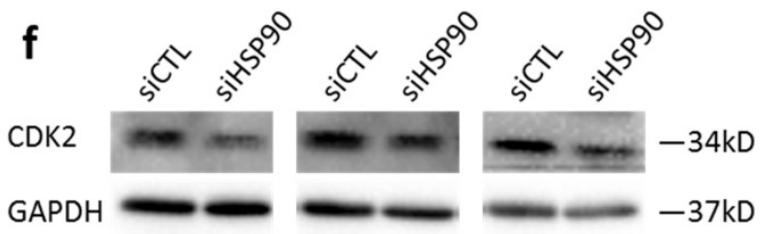

MCF-7/WT MCF-7/ ADM MDA-MB-231

g

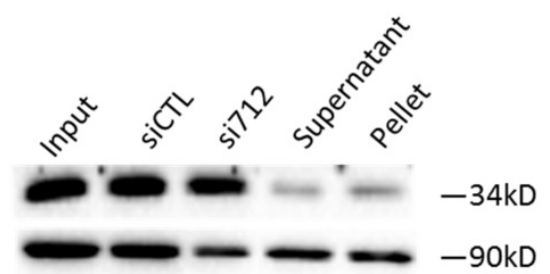

IB:HSP9O

$-90 \mathrm{kD}$

IP:HSP9O

IB:CDK2

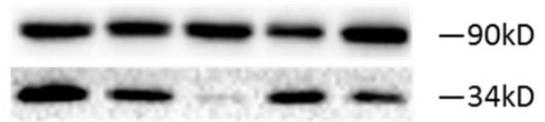

i

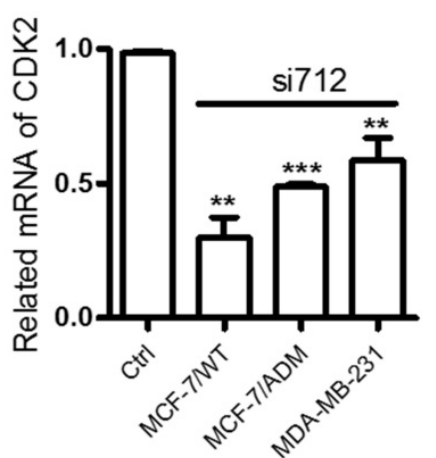

Figure 3. Lnc7 12-HSP90 regulate breast cancer cell proliferation via CDK2 pathway. (a) Cell vitality assays were used to determine the cell viability of Lnc712 siRNA transfected MCF-7, MCF-7/ADM and MDA-MB-231 cells. Results are mean \pm SD of two independent experiments with duplicate wells. ***P <0.001. (b) Flow cytometry was performed to determine the effect of Lnc712 on changes in cell cycle distribution. (c)HSP90 and Cdc37 interaction was studied in MDA-MB-231 cells transfected with Lnc712 siRNA or scrambled siRNA by Co-IP. (d) Hierarchical clustering of distinct mRNAs in MCF-7/ADM and MCF-7/WT cells. Profiling was replicated 3 times. (e) Western blot for CDK2 in three breast cancer cells that transfected with Lnc712 siRNA. (f) Western blot for CDK2 in three breast cancer cells that transfected with HSP90 siRNA.(g) HSP90 and CDK2 interaction was studied in MDA-MB-231 cells transfected with Lnc712 siRNA or scrambled siRNA by Co-IP. (h) Knockdown of Lnc712 increases ubiquitination of CDK2 in MDA-MB-231 cells. (i) CDK2 mRNA level was decreased when cells were treated with Lnc712 siRNA. 
Regarding the action of HSP90 in cell-cycle regulation, cell division cycle 37 (Cdc37) is one of the best-studied co-chaperones of HSP90 [18-21]. As expected, HSP90 co-immunoprecipitated with Cdc37 in MDA-MB-231 cells, while knockdown of Lnc712 significantly decreased the HSP90-Cdc37 interaction (Fig. 3c), suggesting that Lnc712 interacts with HSP90 and reinforces the stability of the HSP90-Cdc37 complex.

Nearly 300 client proteins of HSP90-Cdc37 has been identified. We then calculated the correlation between the expression of lncRNAs and coding genes using the database GSE81971. CDK2, a known client protein of HSP90-Cdc37 [22], was then identified because its expression was strongly positively correlated with high level of Lnc712 (Fig. 3d). To validate, we found CDK2 protein level was higher in breast cancer cells than normal breast cells (Supplementary Fig. 3c). Importantly, the CDK2 protein level was decreased by treatment with either Lnc712 or HSP90 siRNA (Fig. 3e and f). The downregulation of CDK2 protein by Lnc712 might be attributable to ubiquitination of the protein as previous studies suggested that functional HSP90-CDC37 acts to stabilize activate CDK2 [23]. Indeed, we found in Coimmunoprecipitation assay that HSP90-CDK2 interactions was decreased after Lnc712 knockdown (Fig. 3g). Such changes induced by siLnc712 then abolished the stabilization of CDK2 by HSP90-CDC37, and CDK2 was ubiquitinated (Fig. $3 \mathrm{~h})$. The decreased CDK2 protein was accompanied by a reduced level of CDK2 mRNA (Fig. 3i). Together, these results indicate that Lnc712 interacts with HSP90 and controls the activity of CDK2.

Finally, we investigated the effect of Lnc712 on the proliferation of breast cancer cells in vivo. BALB/C mice were used as an in vivo model of mammary carcinoma. The mouse tumor model was generated by intradermal injection of MDA-MB-231 cells. Five mice were treated with Lnc712 siRNA twice a week for 3 consecutive weeks. After 3 weeks, tumors were collected for measurements of tumor size and weight. Tumors treated with Lnc712 siNRA were significantly smaller than control tumors (Fig. 4a and b). The knockdown effects of Lnc712 siRNA were confirmed by measuring the Lnc712 levels (Fig. 4c). Immunohistochemical analyses of randomly-selected xenograft MDA-MB-231 tumors from nude mice demonstrated that tumors with Lnc712 knockdown expressed lower levels of CDK2 (Fig. 4d). Thus, Lnc712 promotes breast cancer cell growth in vivo.

\section{Discussion}

The complete sequencing of the human genome revealed many surprises, and increasing numbers of
lncRNAs have been identified [24]. Researches have demonstrated that lncRNAs participate in various cellular processes, including the regulation of epigenetic signatures, gene expression, and proliferation [25, 26]. In breast cancer, many lncRNAs such as MIAT (myocardial infarction-associated transcript), SNHG1 (small nucleolar RNA host gene 1), and MALAT1 (metastasis-associated lung adenocarcinoma transcript 1) have been reported to participate in the occurrence, progression, and metastasis of tumors [27-29]. This means that lncRNAs are emerging as potential biomarkers for the diagnosis, prognosis, and therapy of breast cancer.

At the beginning of the experiments, we tried to identify chemoresistance-related lncRNAs, and then identified a higher expression of Lnc712 in chemoresistance MCF-7/ADM cells than in chemosensitive MCF-7/WT cells. By seeking the related mechanism, we found Lnc712 directly control the proliferation of MCF-7/ADM cells, making them more vulnerable to the stress of adriamycin. Because 'high proliferative' is universal to all cancer cells regardless their chemo-response, so we then tried to explore the role of Lnc712 in regulating breast cancer cell proliferation and progression. We found that Lnc712, located on chromosome 12, is highly expressed in breast cancer, then investigated its oncogenic role by evaluating its effects on the proliferation of breast cancer cells. We demonstrated that Lnc712 knockdown inhibited proliferation and growth through cell-cycle-arrest in G0/G1. Moreover, we also found that CDK2 protein levels were positively correlated with Lnc712, indicating that Lnc712 regulates the cell cycle by regulating CDK2. CDK2 is critically involved in cell cycling and has been proposed as a potential cancer target [30]. Thus, Lnc712 may play a key role in regulating the development and progression of breast cancer (Fig. 5).

It is well-known that HSP90 is one of the major molecular chaperones in eukaryotic cells [31]. It is an important component of the chaperone machinery, which facilitates protein folding and regulates the stabilization and activity of numerous client proteins [32-34]. HSP90 client proteins originate from distinct functional classes, including transcription factors, kinases, and steroid hormone receptors. Many of these client proteins are commonly overexpressed in cancer cells [35]. Many studies have shown that the formation of IncRNA-protein complexes is an important process by which lncRNAs carry out their functions. An RNA-protein complex can assemble chromatin-modifying complexes [36] and modulate mRNA stability $[37,38]$. Based on our results, Lnc712 functions by forming an RNA-protein complex with HSP90. 
a

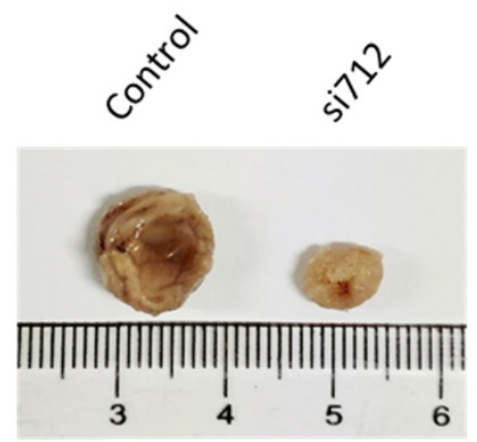

b

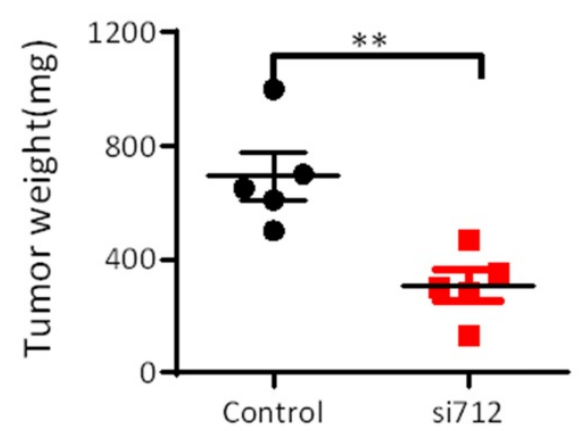

d
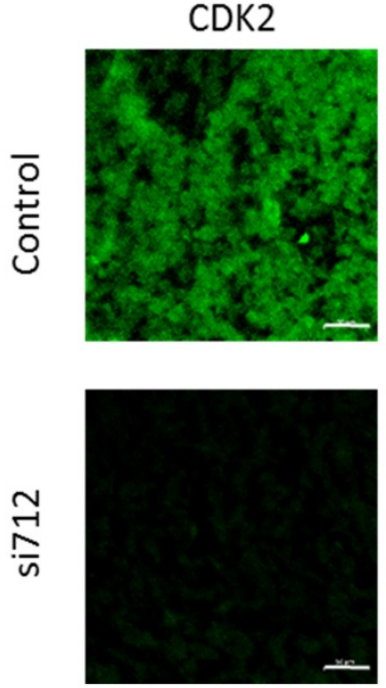

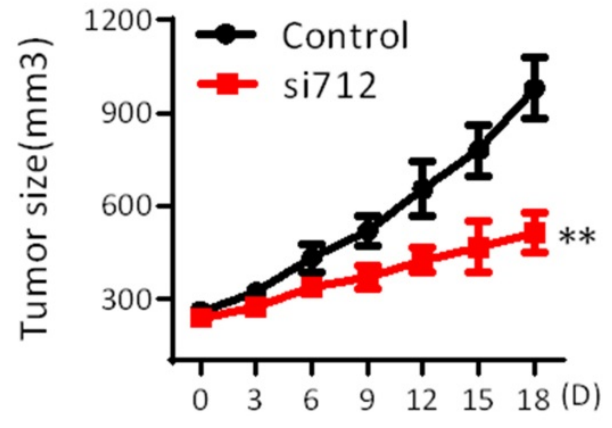

C

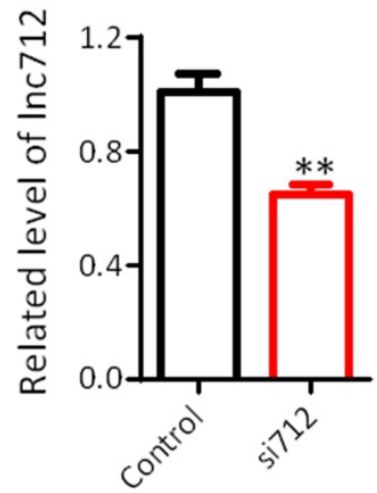

Figure 4. Lnc712 promotes breast cancer cell growth in vivo. (a) Representative figure and Growth curves for the xenograft of Lnc712-knockdown tumor. (b)Tumor weights of the xenograft Lnc712-knockdown tumors were measured. (c) RT-qPCR for Lnc712 level in MDA-MB-231 xenograft. (d) Immunofluorescence images showing the intensity of CDK2 expression in tumors from xenografted mice. scale bar, $50 \mu \mathrm{m}$.

Co-chaperones are key regulators that drive the diverse functions of the HSP90 chaperone machinery. These co-chaperones, including HOP, Cdc37, SGTA, TAH1, and p23, in concert with HSP90 form complexes that direct a broad range of specific clients to HSP90 [32]. Cdc37 is the best-studied of these co-chaperones, and is considered to be exclusively associated with protein kinases [39]. Recent progress has allowed the interactions of HSP90-Cdc37-client protein to be determined $[19,40]$. To date, nearly 300 client proteins of HSP90-Cdc37 have been found, and CDK2 is one of them [22]. Our results show that Lnc712 reinforces the interactions of HSP90 and Cdc37 to regulate CDK2 expression. 


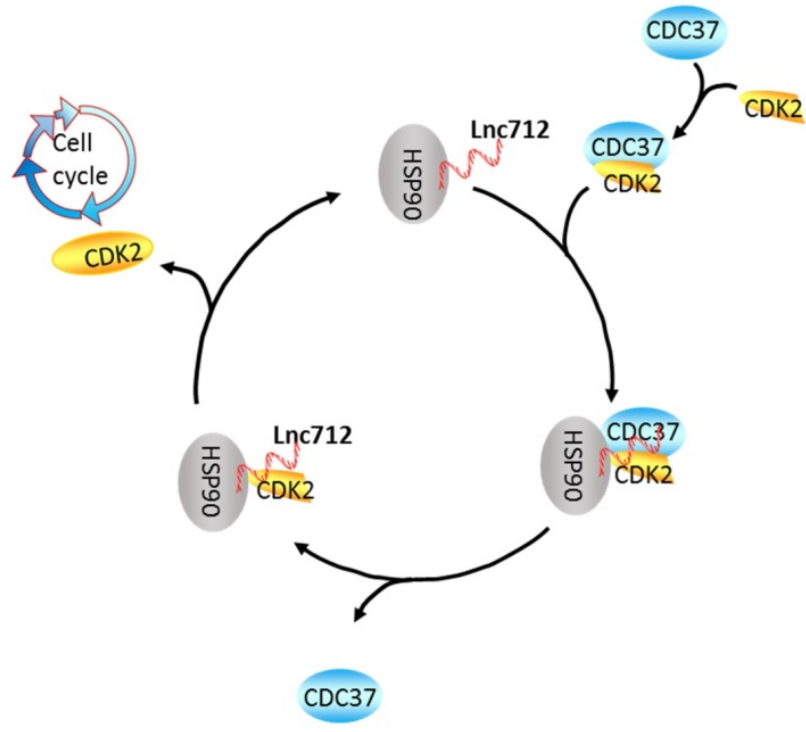

Figure 5. A speculative model of the Lnc712-HSP90-Cdc37 cycle. Cdc37 first establishes connection with the CDK2 to create a Cdc37-CDK2 complex. Then, the binary complex binds to Hsp90-Lnc712 to form a stable complex. The formation of the Hsp90-Cdc37-CDK2 complex finally facilitates CDK2 maturation. Lnc712 helps HSP90 to participate in the interaction with Cdc37 and CDK2.

In recent years, HSP90 has become a potential target for a number of diseases including cancer, neurodegeneration, and infectious diseases [41-44]. The development of HSP90 inhibitors has grown rapidly. For example, 17-DMAG binds to HSP90 and inhibits its function, which eventually results in the degradation of HSP90 client proteins [45]; KU675, a novel C-terminal HSP90 inhibitor, has potent anti-proliferative and cytotoxic activity along with client protein degradation, without the induction of a heat-shock response in both androgen-dependent and -independent prostate cancer cell lines [46]; and triptolide inhibits HSP90 $\beta$ in a three-fold manner to decrease the CDK4 protein levels in HeLa cells, causing a reduction in the phosphorylation of $\mathrm{Rb}$, and resulting in cell-cycle-arrest at G1 [47]. So far, none of the inhibitors of HSP90 have been approved for clinical application in cancer, because systematic inhibition of HSP90 activity may lead to high cytotoxicity mainly due to dysfunction in heat-shock responses [48, 49]. However, our research may provide new strategy for inhibiting cancerous HSP90 by targeting Lnc712, which is specifically highly expressed in breast cancer cells but not in surrounding healthy tissues. In future works, it is worth investigating the expression of Lnc712 in different breast cancer subtypes, and also in other types of cancers to acquire better understanding of Lnc712 as an anti-cancer target.

Our results reveal a novel therapeutic target for human breast cancer. Destabilizing the HSP90-Cdc37 complex to arrest the cell-cycle by reducing the binding of Lnc712 to HSP90 is a new approach.

\section{Supplementary Material}

Supplementary figures and tables. http://www.ijbs.com/v16p0162s1.pdf

\section{Acknowledgment}

This work was supported by the National Natural Science Foundation of China (81572940, 81870362), Fundamental Research Funds for the Central Universities (JUSRP51615B). We also thanks the support from innovative research team grant from Taizhou local government (1801GY17) to J. Xue in Yeda research institute of gene and cell therapy.

\section{Author contributions}

Y.C, C.L., Z.Z. and A.M. performed experiments with the help from D.H., X.M., F.G., L.F. and L.F.. D.H. and X.M. designed experiments. Y.C. and D.H. did the statistical analysis. Y.C. and D.H. wrote the manuscript. All the authors have read and approve this manuscript.

\section{Competing Interests}

The authors have declared that no competing interest exists.

\section{References}

1. Mollard S, Ciccolini J, Imbs DC, El Cheikh R, Barbolosi D, Benzekry S. Model driven optimization of antiangiogenics + cytotoxics combination: application to breast cancer mice treated with bevacizumab + paclitaxel doublet leads to reduced tumor growth and fewer metastasis. Oncotarget. 2017; 8: 23087-98.

2. Ferlay J, Soerjomataram I, Dikshit R, Eser S, Mathers C, Rebelo M, et al. Cancer incidence and mortality worldwide: sources, methods and major patterns in GLOBOCAN 2012. International journal of cancer. 2015; 136: E359-86.

3. Brown CJ, Hendrich BD, Rupert JL, Lafreniere RG, Xing Y, Lawrence J, et al. The human XIST gene: analysis of a $17 \mathrm{~kb}$ inactive X-specific RNA that contains conserved repeats and is highly localized within the nucleus. Cell. 1992; 71: 527-42.

4. Bu D, Yu K, Sun S, Xie C, Skogerbo G, Miao R, et al. NONCODE v3.0: integrative annotation of long noncoding RNAs. Nucleic acids research. 2012; 40: D210-5.

5. Feng J, Bi C, Clark BS, Mady R, Shah P, Kohtz JD. The Evf-2 noncoding RNA is transcribed from the Dlx-5/6 ultraconserved region and functions as a Dlx-2 transcriptional coactivator. Genes \& development. 2006; 20: 1470-84.

6. Martianov I, Ramadass A, Serra Barros A, Chow N, Akoulitchev A. Repression of the human dihydrofolate reductase gene by a non-coding interfering transcript. Nature. 2007; 445: 666-70.

7. Wang X, Arai S, Song X, Reichart D, Du K, Pascual G, et al. Induced ncRNAs allosterically modify RNA-binding proteins in cis to inhibit transcription. Nature. 2008; 454: 126-30.

8. Rajan KS, Ramasamy S, George-William JN, Rajendhran J. Emerging cardiac non-coding landscape: The importance of meta-analysis. Biochimie. 2017; 133: 87-94.

9. Marcucci G, Mrozek K, Radmacher MD, Garzon R, Bloomfield CD. The prognostic and functional role of microRNAs in acute myeloid leukemia. Blood. 2011; 117: 1121-9.

10. Guo H, Zhao L, Shi B, Bao J, Zheng D, Zhou B, et al. GALNT5 uaRNA promotes gastric cancer progression through its interaction with HSP90. Oncogene. 2018; 37: 4505-17.

11. Ma X, Cai Y, He D, Zou C, Zhang P, Lo CY, et al. Transient receptor potential channel TRPC5 is essential for P-glycoprotein induction in drug-resistant cancer cells. ProcNatlAcadSciUSA. 2012; 109: 16282-7.

12. Ma X, Chen Z, Hua D, He D, Wang L, Zhang P, et al. Essential role for TrpC5-containing extracellular vesicles in breast cancer with chemotherapeutic resistance. ProcNatlAcadSciUSA. 2014

13. Goda K, Bacso Z, Szabo G. Multidrug resistance through the spectacle of P-glycoprotein. CurrCancer Drug Targets. 2009; 9: 281-97. 
14. Traverso N, Ricciarelli R, Nitti M, Marengo B, Furfaro AL, Pronzato MA, et al. Role of glutathione in cancer progression and chemoresistance. OxidMedCell Longev. 2013; 2013: 972913.

15. Mayer MP, Le Breton L. Hsp90: breaking the symmetry. Mol Cell. 2015; 58: 8-20.

16. Tsutsumi S, Neckers L. Extracellular heat shock protein 90: a role for a molecular chaperone in cell motility and cancer metastasis. Cancer Sci. 2007; 98: 1536-9.

17. Vahid S, Thaper D, Zoubeidi A. Chaperoning the Cancer: The Proteostatic Functions of the Heat Shock Proteins in Cancer. Recent Pat Anticancer Drug Discov. 2017; 12: 35-47.

18. Wang L, Li L, Zhou ZH, Jiang ZY, You QD, Xu XL. Structure-based virtual screening and optimization of modulators targeting Hsp90-Cdc37 interaction. European journal of medicinal chemistry. 2017; 136: 63-73.

19. Taipale M, Krykbaeva I, Koeva M, Kayatekin C, Westover KD, Karras GI, et al. Quantitative analysis of HSP90-client interactions reveals principles of substrate recognition. Cell. 2012; 150: 987-1001.

20. Calderwood SK. Cdc37 as a co-chaperone to Hsp90. Sub-cellular biochemistry. 2015; 78: 103-12.

21. Gray PJ, Jr., Prince T, Cheng J, Stevenson MA, Calderwood SK. Targeting the oncogene and kinome chaperone CDC37. Nature reviews Cancer. 2008; 8: 491-5.

22. Prince T, Sun L, Matts RL. Cdk2: a genuine protein kinase client of Hsp90 and Cdc37. Biochemistry. 2005; 44: 15287-95.

23. Jha KN, Coleman AR, Wong L, Salicioni AM, Howcroft E, Johnson GR. Heat shock protein 90 functions to stabilize and activate the testis-specific serine/threonine kinases, a family of kinases essential for male fertility. J Biol Chem. 2013; 288: 16308-20.

24. Derrien T, Johnson R, Bussotti G, Tanzer A, Djebali S, Tilgner H, et al. The GENCODE v7 catalog of human long noncoding RNAs: analysis of their gene structure, evolution, and expression. Genome Res. 2012; 22: 1775-89.

25. Xiong Y, Wang L, Li Y, Chen M, He W, Qi L. The Long Non-Coding RNA XIST Interacted with MiR-124 to Modulate Bladder Cancer Growth, Invasion and Migration by Targeting Androgen Receptor (AR). Cellular physiology and biochemistry : international journal of experimental cellular physiology, biochemistry, and pharmacology. 2017; 43: 405-18.

26. Song W, Zou SB. Prognostic role of IncRNA HOTAIR in esophageal squamous cell carcinoma. Clinica chimica acta; international journal of clinical chemistry. 2016; 463: 169-73.

27. Almnaseer ZA, Mourtada-Maarabouni M. Long non-coding RNA MIAT regulates apoptosis and the apoptotic response to chemotherapeutic agents in breast cancer cell lines. Bioscience reports. 2018.

28. Pei $X$, Wang $X$, Li H. LncRNA SNHG1 regulates the differentiation of Treg cells and affects the immune escape of breast cancer via regulating miR-448/IDO. International journal of biological macromolecules. 2018; 118: 24-30.

29. Wang Z, Katsaros D, Biglia N, Shen Y, Fu Y, Loo LWM, et al. High expression of long non-coding RNA MALAT1 in breast cancer is associated with poor relapse-free survival. Breast cancer research and treatment. 2018.

30. Soffar A, Storch K, Aleem E, Cordes N. CDK2 knockdown enhances head and neck cancer cell radiosensitivity. Int J Radiat Biol. 2013; 89: 523-31.

31. Whitesell L, Lindquist SL. HSP90 and the chaperoning of cancer. Nat Rev Cancer. 2005; 5: 761-72

32. Schopf FH, Biebl MM, Buchner J. The HSP90 chaperone machinery. Nature reviews Molecular cell biology. 2017; 18: 345-60.

33. Zhao R, Davey M, Hsu YC, Kaplanek P, Tong A, Parsons AB, et al. Navigating the chaperone network: an integrative map of physical and genetic interactions mediated by the hsp90 chaperone. Cell. 2005; 120: 715-27.

34. Trepel J, Mollapour M, Giaccone G, Neckers L. Targeting the dynamic HSP90 complex in cancer. Nature reviews Cancer. 2010; 10: 537-49.

35. Young JC, Agashe VR, Siegers K, Hartl FU. Pathways of chaperone-mediated protein folding in the cytosol. Nature reviews Molecular cell biology. 2004; 5: 781-91.

36. Klattenhoff CA, Scheuermann JC, Surface LE, Bradley RK, Fields PA, Steinhauser ML, et al. Braveheart, a long noncoding RNA required for cardiovascular lineage commitment. Cell. 2013; 152: 570-83.

37. Kretz M, Siprashvili Z, Chu C, Webster DE, Zehnder A, Qu K, et al. Control of somatic tissue differentiation by the long non-coding RNA TINCR. Nature. 2013; 493: 231-5

38. Liu X, Li D, Zhang W, Guo M, Zhan Q. Long non-coding RNA gadd7 interacts with TDP-43 and regulates Cdk6 mRNA decay. EMBO J. 2012; 31: 4415-27.

39. Siligardi G, Panaretou B, Meyer P, Singh S, Woolfson DN, Piper PW, et al. Regulation of Hsp90 ATPase activity by the co-chaperone Cdc37p/p50cdc37. The Journal of biological chemistry. 2002; 277: 20151-9.

40. Keramisanou D, Aboalroub A, Zhang Z, Liu W, Marshall D, Diviney A, et al. Molecular Mechanism of Protein Kinase Recognition and Sorting by the Hsp90 Kinome-Specific Cochaperone Cdc37. Molecular cell. 2016; 62: 260-71.

41. Paul S, Mahanta S. Association of heat-shock proteins in various neurodegenerative disorders: is it a master key to open the therapeutic door? Mol Cell Biochem. 2014; 386: 45-61.

42. Hong DS, Banerji U, Tavana B, George GC, Aaron J, Kurzrock R. Targeting the molecular chaperone heat shock protein 90 (HSP90): lessons learned and future directions. Cancer treatment reviews. 2013; 39: 375-87.

43. Veri A, Cowen LE. Progress and prospects for targeting Hsp90 to treat fungal infections. Parasitology. 2014; 141: 1127-37.
44. Gillan V, Devaney E. Nematode Hsp90: highly conserved but functionally diverse. Parasitology. 2014; 141: 1203-15.

45. Mellatyar H, Talaei S, Pilehvar-Soltanahmadi Y, Barzegar A, Akbarzadeh A, Shahabi A, et al. Targeted cancer therapy through 17-DMAG as an Hsp90 inhibitor: Overview and current state of the art. Biomedicine \& pharmacotherapy = Biomedecine \& pharmacotherapie. 2018; 102: 608-17.

46. Liu W, Vielhauer GA, Holzbeierlein JM, Zhao H, Ghosh S, Brown D, et al KU675, a Concomitant Heat-Shock Protein Inhibitor of Hsp90 and Hsc70 that Manifests Isoform Selectivity for Hsp90alpha in Prostate Cancer Cells. Molecular pharmacology. 2015; 88: 121-30.

47. Zhang FZ, Ho DH, Wong RH. Triptolide, a HSP90 middle domain inhibitor, induces apoptosis in triple manner. Oncotarget. 2018; 9: 22301-15.

48. Pedersen KS, Kim GP, Foster NR, Wang-Gillam A, Erlichman C, McWilliams RR. Phase II trial of gemcitabine and tanespimycin (17AAG) in metastatic pancreatic cancer: a Mayo Clinic Phase II Consortium study. Investigational new drugs. 2015; 33: 963-8.

49. Acquaviva J, He S, Sang J, Smith DL, Sequeira M, Zhang C, et al. mTOR inhibition potentiates HSP90 inhibitor activity via cessation of HSP synthesis. Molecular cancer research : MCR. 2014; 12: 703-13. 